\title{
Imaging, biopsy and non-surgical treatment of thyroid lesions: where are we at?
}

\author{
Alain M. Lavoipierre ${ }^{1}$ (D) \\ Received: 27 April 2020 / Revised: 8 June 2020 / Accepted: 23 July 2020 / Published online: 4 August 2020 \\ (C) European Society of Radiology 2020
}

\section{Key Points}

- Ultrasound is the prevalent modality for assessment of the thyroid and biopsy guidance of lesions.

- The various TI-RADS classifications provide scoring systems which help indicate the likelihood of malignancy.

- Ongoing research helps to improve the characterisation of nodules, biopsy techniques and non-surgical treatment methods.

Imaging, biopsy and non-surgical management of thyroid lesions frequently present challenges, as the thyroid is a relatively small gland where lesions often abut on to key structures, such as the carotid artery, compounded by mimickers of real abnormalities. Ultrasound remains the prevalent modality for thyroid assessment, with rapid improvements in equipment, complemented by experience garnered by sonographers and sonologists.

While categorizing diffuse thyroid diseases can, at times, be problematic, in diagnosing thyroid nodules, the TI-RADS (Thyroid Imaging Reporting and Data System) classification in its different forms, including ACR TI-RADS (American College of Radiology, 2017) [1], EU-TIRADS (European Thyroid Association, 2017) [2], K-TIRADS (Korean Society of Thyroid Radiology, 2016) [3] and the ATA (American Thyroid Association) guidelines [4], mainly through scoring systems, has brought considerable rationale to the classification of nodules. The scores attributed to nodules through ultrasound help determine the likelihood of malignancy. The higher the TI-RADS level, the greater the likelihood of malignancy, thereby necessitating tissue sampling.

Once the potential nature of a thyroid lesion is established, obtaining tissue for diagnostic purposes in appropriate cases can be achieved either through fine needle aspiration cytology (FNAC), or by core needle biopsy (CNB). While FNAC is recommended as the first-choice method to diagnose thyroid

Alain M. Lavoipierre

alainl@bigpond.net.au

1 Lake Imaging Specialist and Research Centre, Level 5, 14 Blackwood St., North Melbourne, Vic 3051, Australia nodules, $\mathrm{CNB}$ has been suggested as an alternative to repeating FNAC in non-diagnostic or equivocal results. For those who require FNAC, the patients can, as we do in our institution, be "grouped" in one session with a cytopathologist present, who then immediately examines the slides obtained, thus providing the opportunity to repeat FNAC immediately and improve diagnostic yield.

Paja et al evaluated the performance of CNB in thyroid, used as first choice for biopsy. They concluded that CNB was accurate and had few complications, as well as a low rate of non-diagnostic and indeterminate diagnoses. It can also be an alternative method when FNAC performs poorly. In a series which included $4412 \mathrm{CNB}$, for follicular lesions and malignancy (both leading to recommendation for surgery), sensitivity was $96 \%$ and specificity was $93.7 \%$. Repeat CNB in 53 insufficient biopsies obtained 50 diagnostic results. The authors used $18 \mathrm{G}$ needles and had a minor complication rate of $2.2 \%$ and major complications in $0.09 \%$. [5, 6].

A different approach was taken by $\mathrm{Ha}$ et al to assess the malignancy risk of initially benign thyroid nodules, which they validated with various TI-RADS guidelines. They investigated the malignancy rate and probability by using various malignancy stratification systems in initially biopsy-proven benign nodules, seeking to determine their clinical relevance in the management of benign thyroid nodules. They analyzed 2747 (229 malignant and 2518 benign) thyroid nodules diagnosed by initial biopsy with $28.2 \pm 9.1$ months of follow-up. The malignancy probability of nodules was calculated by using various malignancy risk stratification systems. The overall calculated malignancy rate was $8.3 \%$ (229 of 2747). Initially biopsy-proven benign nodules exhibited a $\leq$ malignancy probability when assessed as "low suspicion" 
according to several TI-RADS guidelines. They concluded that when biopsy-proven benign nodules exhibit a "low suspicion" US pattern and low malignancy probability as stratified by various TIRADS guidelines, imaging surveillance instead of a second biopsy is warranted [7].

The quest for endeavouring to characterize nodules as benign or malignant on ultrasound criteria continues unabated. Lee et al in a recent paper attempted to stratify the US malignancy risk of thyroid nodules based on their degree of hypoechogenicity and echotexture. They looked at 2255 nodules $(\geq 1 \mathrm{~cm})$ with final diagnoses (malignancy rate, $13 \%$ ) and found no significant difference of malignancy risk between the homogeneously markedly hypoechoic and moderately hypoechoic nodules. However, the malignancy risks of markedly and moderately hypoechoic nodules were significantly higher than those of mildly hypoechoic nodules. Heterogeneous predominantly hypoechoic nodules showed a significantly higher malignancy risk than predominantly iso- or hyperechoic thyroid nodules. There were no significant differences of malignancy risk between heterogeneous predominantly hypoechoic and homogeneous hypoechoic nodules according to the degree of hypoechogenicity and between heterogeneous predominantly iso- or hyperechoic nodules and homogeneous iso- or hyperechoic nodules [8].

The echogenicity of thyroid texture is a crucial marker in the assessment of nodules but also of diffuse thyroid disease [9]. Good sonographic equipment is vital and optimal training of sonographers and sonologists is equally important. Another paper by Lee et al deals with the prognostic value of acoustic structure quantification (ASQ) in patients with Hashimoto's thyroiditis. They surveyed 90 patients for the development of overt hypothyroidism over a median period of 40 months. ASQ were dichotomized based on optimal cutoff values obtained from ROC curve analysis. The cumulative rate of overt hypothyroidism was $67.7 \%$. Qualitative echogenicity between groups was of little value. However, the ASQ average, standard deviation and focal disturbance ratio were associated with an increased risk of overt hypothyroidism [10].

The value of ultrasound in imaging, biopsy and treatment of thyroid conditions cannot be underestimated. As an example, Lang et al have looked at 2-year outcomes of singlesession high-intensity focused ultrasound (HIFU) treatment in persistent or relapsed Graves' disease in 75 patients, $84 \%$ of whom were females. They received HIFU ablation of the central portion of each thyroid lobe, with areas near the trachea-oesophageal groove and common carotid artery unablated. They were followed for at least 24 months. Baseline TSH, freeT4, anti-thyroid antibodies, and TSH receptor (TSHR) antibodies were checked. Primary outcome was the 24-month relapse rate, with relapse referring to hyperthyroidism. The 24 -month relapse rate was $41.3 \%$. No patient suffered from hypothyroidism. Three patients suffered from temporary vocal cord palsy, but they recovered spontaneously after 2 months. In univariate analysis, higher daily rate of carbimazole and higher baseline TSHR level were significant factors for disease relapse. In the multivariate analysis, higher baseline TSHR level was a significant independent factor for disease relapse [11].

In conclusion, the importance of sonographic assessment of the thyroid to diagnose, aid tissue sampling and help manage conditions (both diffuse and nodular) is improving almost exponentially.

Funding information The authors state that this work has not received any funding.

\section{Compliance with ethical standards}

Guarantor The scientific guarantor of this publication is Alain Lavoipierre.

Conflict of interest The author of this manuscript declares no relationships with any companies, whose products or services may be related to the subject matter of the article.

Statistics and biometry No complex statistical methods were necessary for this paper.

Informed consent Written informed consent was not required for this study because this work is an Editorial.

Ethical approval Institutional Review Board approval was not required because this work is an Editorial.

\section{Methodology}

- retrospective

- observational

\section{References}

1. Tessler FN, Middleton WD, Grant EG et al (2017) ACR Thyroid Imaging, Reporting and Data System (TI-RADS): white paper of the ACR TI-RADS Committee. J Am Coll Radiol 14(5):587-595

2. Russ G, Bonnema SJ, Erdogan MF, Durante C, Ngu R, Leenhardt L (2017) European Thyroid Association guidelines for ultrasound malignancy risk stratification of thyroid nodules in adults: the EU TI-RADS. Eur Thyroid J 6:225-237

3. Russ G, Bonnema SJ, Erdogan MF, Durante C, Ngu R, Leenhardt L (2016) Ultrasonography diagnosis and imaging-based management of thyroid nodules: revised Korean Society of Thyroid Radiology Consensus Statement and Recommendations. Korean J Radiol 17(3):370-395

4. Haugen BR, Alexander EK, Bible KC et al (2016) 2015 American Thyroid Association management guidelines for adult patients with thyroid nodules and differentiated thyroid cancer. Thyroid 26(1):1133

5. Paja M, del Cura JL, Zabala R, Korta I, Ugalde A, López JI (2019) Core-needle biopsy in thyroid nodules: performance, accuracy and complications. Eur Radiol 29:4889-4896 
6. Chung SR, Suh CH, Baek JH, Choi YJ, Lee JH (2018) The role of core needle biopsy in the diagnosis of initially detected thyroid nodules: a systematic review and meta-analysis. Eur Radiol 28(11):4909-4918

7. Ha SM, Baek JH, Choi YJ et al (2019) Malignancy risk of initially benign thyroid nodules: validation with various Thyroid Imaging Reporting and Data System guidelines. Eur Radiol 29:133-140

8. Lee JY, Dong GN, Yoon SJ et al (2020) Ultrasound malignancy risk stratification of thyroid nodules based on the degree of hypoechogenicity and echotexture. Eur Radiol 30:1653-1663

9. Yuen HY, Wong KT, Ahuja AT (2016) Sonography of diffuse thyroid disease. AJUM 19(1):13-19
10. Lee JY, Hong HS, Kim CH (2019) Prognostic value of acoustic structure quantification in patients with Hashimoto's thyroiditis. Eur Radiol 29:5971-5980

11. Lang BH-H, Woo Y-C, Chiu KW-H (2019) Two-year outcomes of single session high-intensity focused ultrasound (HIFU) treatment in persistent or relapsed Graves' disease. Eur Radiol 29:6690-6698

Publisher's note Springer Nature remains neutral with regard to jurisdictional claims in published maps and institutional affiliations. 